\title{
The Emerging Role of Multiplex Tandem Mass Spectrometry Analysis for Therapeutic Drug Monitoring and Personalized Medicine
}

\author{
Decosterd $\mathrm{LA}^{1^{*}}$, Widmer $\mathrm{N}^{2,4^{*}}$, André $\mathrm{P}^{2}$, Aouri $\mathrm{M}^{2,3}$, Buclin $\mathrm{T}^{2}$ \\ ${ }^{1}$ Laboratory and ${ }^{2}$ Division of Clinical Pharmacology, Service of Biomedicine \\ ${ }^{3}$ Laboratory of Clinical Chemistry, Service of Biomedicine
}

Centre Hospitalier Universitaire Vaudois and University of Lausanne, Switzerland

${ }^{4}$ Pharmacy of Eastern Vaud Hospitals, Vevey, Switzerland

* contributed equally to the article

Submitted to:

Trends in Analytical Chemistry Special Issues: "Mass spectrometry in clinical routine modern analytical chemistry in laboratory medicine"

\section{Correspondence:}

Prof Laurent A. Decosterd, PhD

Laboratory of clinical Pharmacology

Service of Biomedicine, BH18 - Lab 218

Centre Hospitalier Universitaire Vaudois and University of Lausanne

$\mathrm{CH}-1011$ Lausanne Switzerland

Phone: +4121314 4272

Fax: $\quad+41213148098$

E-mail: LaurentArthur.Decosterd@chuv.ch 


\begin{abstract}
Liquid chromatography coupled to tandem mass spectrometry (LC-MS/MS) constitutes now a key instrumental component of medical laboratory centres and has greatly facilitated the development of a considerable number of drugs assays for Therapeutic Drug Monitoring (TDM). The concept of TDM implies the measurement of concentrations of certain drugs in patients' blood, followed by dosage individualization based on patients' circulating exposure, targeting a pre-defined concentration range. This approach aims at increasing the likelihood of achieving therapeutic efficacy, while reducing the risk of toxicity. TDM can contribute to the rational individualization of critical drug treatments, notably antivirals, antibiotics, antiepileptics, psychotropic drugs and targeted anticancer agents, in the context of growing efforts towards personalized medicine. This article aims at reviewing the basic concepts, the role and the perspectives of evolution of TDM. It presents selected examples of clinical applications and gives some insights on how TDM is definitely benefiting from the tremendous development of LC-MS/MS techniques.
\end{abstract}

\title{
Key words
}

Liquid Chromatography coupled to tandem Mass Spectrometry; Therapeutic Drug Monitoring; Drug Dosage Individualisation; Precision Medicine 


\section{Introduction}

Over the past 15 years, the technique of liquid chromatography coupled to tandem mass spectrometry (LC-MS/MS) has revolutionized the field of bioanalysis and has progressively emerged as a key instrumental component for the daily practice of laboratory medicine. LC-MS/MS has strikingly facilitated the development of assays for a considerable number of analytes, not to mention its impact on the organisational work flow of the laboratory and turn-around-time (i.e. TAT, the delay before a result is available for interpretation after sample receipt at the laboratory) [1-6].

It has been established over the last decades that the therapeutic use of some drugs could be optimized by an individualization of their dosage, based on circulating (blood or plasma) concentrations measurement in the patients. This approach specifically corresponds to the concept of Therapeutic Drug Monitoring (TDM). In the context of TDM, a tremendous number of assays by LCMS/MS, especially triple quadrupole tandem MS, have been developed over the last years (nearly 6000 hits retrieved on PubMed about "mass spectrometry" and "therapeutic drug monitoring" as of October 30, 2015). A vast array of drugs can nowadays be measured in blood and plasma, and in other biological fluids (urine, saliva, cerebrospinal fluid, dried blood spots, cells and tissues extracts, etc.), reflecting the growing interest of mass spectrometry approaches in the field of TDM. The facilitated access to stable isotopically-labelled internal standards for most drugs has been instrumental for the rapid development and validation of LC-MS/MS methods, and has provided a further step toward the general acceptance of mass spectrometry approaches in clinical laboratories, as it can compensate for the variability of matrix effects, to which LC-MS/MS is vulnerable. Because of the unprecedented levels of selectivity and sensitivity achieved by LC-MS/MS, the analytical development by specialized laboratories is nowadays no more the limiting ("bottleneck") step that restricts the potential implementation of TDM for any given drug. At present, the critical issue is rather to interpret the analytical results and to provide clinicians with validated interpretations of concentration data that can be translated into sound dosage adaptation decisions. These aspects of clinical pharmacokinetics and TDM interpretations are generally neglected among bioanalytical scientists, who tend to rather focus on technological aspects of their assays methods.

The present article aims therefore at i) providing a review of the basic concepts, the role and the evolutionary potential of Therapeutic Drug Monitoring (TDM) to readers that may not be familiar with the field of clinical diagnostics, ii) illustrating these concepts by meaningful examples of recent developments, and iii) giving some insights on how the TDM has greatly benefited from the tremendous development of liquid chromatography coupled to tandem mass spectrometry.

For addressing various practical aspects of TDM, we propose to follow the sequential steps of reflexions and actions that are triggered when a drug measurement request is addressed to the medicine laboratory. This review is mainly based on the experience that we have accumulated over the years with our TDM service and on our longstanding expertise in the development of new TDM 
approaches and tools for the optimization of critical treatments involving drugs known for significant between-patient variability.

\section{What is TDM?}

For most systemic therapies, circulating blood (or plasma) drug concentration exposure is recognized as the leading factor associated with pharmacological or toxic effects. Actually, the drug concentration in blood, rather than the dose administered, is closely related to the exposure at the site of pharmacological action, and therefore represents a better predictor than dosage for therapeutic response and/or clinical toxicity. TDM, which might more properly be called "Therapeutic concentration monitoring", exploits the fact that dose-concentration relationships (i.e. the pharmacokinetic step of drug action, "what the body does to the drug") are often more variable than concentration-effect relationships (i.e. the pharmacodynamic step of drug action, "what the drug does to the body"). Multiple sources of variability have been identified, including demographic, environmental, clinical and (pharmaco)genetic factors. The basic concept of TDM implies the measurement of concentration of a drug in the plasma (blood) from a patient under treatment, followed by the rational interpretation of the result and finally by the drug dosage individualization, targeting a pre-defined concentration range. This approach aims at increasing the likelihood of achieving therapeutic efficacy in an individual patient, while reducing the risk of meeting toxicity. TDM thus represents one of the most advanced developments in the growing field of personalized medicine.

\section{What is TDM for?}

The medical rationale behind the concept of TDM is driven by clinical questions that information on circulating drug levels in patients can help to answer. In practice, the majority of long-term treatments deserves to be monitored by one way or another (e,g, blood glucose and glycated haemoglobin $\mathrm{HbA1c}$ in diabetes treatment, which is then simply called "therapeutic monitoring"). When good pharmacodynamic markers for a given treatment are missing, the monitoring of drug levels can represent a useful surrogate, helping at achieving a suitable level of circulating exposure in terms of both effectiveness and safety. More generally, TDM should be considered in case of lack of early or easily accessible pharmacodynamic biomarkers of therapeutic action. Practically, the circulating concentration measured can be considered as the best index of systemic exposition to drug treatment. Table 1 illustrates various concrete reasons for which a blood level measurement can be considered by practitioners.

\section{Which drugs are candidates for TDM?}

Not every drug that is administered to patients is susceptible to benefit from concentration-based dosage adjustment in terms of improvement of therapeutic effect and/or of tolerance profile. For a drug being candidate to TDM, a number of criteria have to be met. First, a precise and accurate analytical method with minimal TAT must be available, allowing timely delivery of quantitative result, ideally on a real-time basis, or at least before the next administered dose. Multiplex analysis allowing to analyse up to 15-20 drugs in the same run are now available thanks to LC-MS/MS technology, and have proved to be invaluable to that endeavour (see below). Secondly, TDM is generally considered 
for drugs that have to be administered on the long term (chronic conditions, e.g. new oral targeted anticancer drugs, antiviral agents against HIV infection, etc.). However, TDM can be also considered for drugs constituting last resort treatments administered to critically ill patients, such as in intensive care units where appropriate exposure is key for maximizing efficacy while minimizing toxicity (e.g. voriconazole for invasive fungal infections [7] and the antibiotic colistin against life-threatening multiresistant Pseudomonas infections [8]). One of the main criteria making a drug a suitable candidate to TDM is a significant inter-individual pharmacokinetic variability (i.e. the drug dosage administered to a given individual is a poor predictor of its systemic exposure), along with a limited intra-patient pharmacokinetic variability (i.e. a drug dosage in a given patient yields similar blood concentrations when measurements are repeated). Finally, before TDM can be considered for a given drug, consistent relationships between blood concentrations and clinical efficacy and/or toxicity must have been demonstrated, and target therapeutic intervals been established and validated clinically. TDM is especially advised for drugs characterized by a narrow therapeutic range (small difference between therapeutic and toxic blood concentration levels). All the above criteria restrict therefore the number of drugs that are formally candidate to a TDM program.

This TDM-guided feedback strategy to adjust the concentration exposure has now become current practice for optimizing the therapeutic use of critical drugs such as selected antibiotics, antiepileptics, immunosuppressants, antifungals and anti-HIV drugs [9]. Despite limited clinical validation, TDM of antiretrovirals is now commonly considered in case of virological failure, adverse drug reactions, drug interaction problems, and special clinical conditions (pregnancy, pediatrics, liver failure etc.) [10]. Similarly, novel oral targeted anticancer drugs also constitute ideal candidates for TDM [11]. The various therapeutic classes presently recognized to benefit from TDM are listed in Table 2 [12].

It must be acknowledged that those consensual criteria for TDM are by far not met for all drugs that can be assayed (selected examples listed in Table 2). On the other hand, randomized controlled studies having formally demonstrated the clinical usefulness of TDM in fine remain rather infrequent. However, we are witnessing significant developments in this area, as user-friendly tools are appearing that facilitate the interpretation of drug levels for users having only basic knowledge of clinical pharmacokinetics, which will likely allow a more rational use of TDM in the medical community (see below).

\section{When and how to collect a blood sample for TDM measurement?}

Endogenous metabolites and bioanalytes commonly measured in medicine laboratories are generally measured in a fasting state, or if not otherwise indicated, at unselected time in patients (except in rare instances such as oral glucose challenge or endocrinology tests). By contrast, time does largely matter when considering TDM measurements (Figure 1), since the blood concentration expected after drug intake oscillates over the dosing interval (time span between two successive drug doses), reaching a maximum ("peak", $\mathrm{C}_{\max }$ ) level (i.e. when drug disposition overrides absorption) followed by a progressive decrease (driven by body clearance capacity, and reflected by drug's plasma half-lives) down to a minimum ("trough", $\mathrm{C}_{\mathrm{min}}$ ) blood level, just prior to the next dose. Most of therapeutic 
intervals considered in clinical practice are in fact trough concentration intervals. In case of significantly earlier or later sampling, TDM interpretation may require a pharmacokinetic extrapolation, as described below. Thus, a drug measurement for TDM imposes a number of constraints on when and how the blood is taken from patients. The principal precautions to observe are listed in Table 3.

\section{How to interpret a TDM measurement?}

After analytical validation, the drug level measurement result is sent to the physicians (example: 3250 $\mu \mathrm{g} / \mathrm{L}$ ) along with therapeutic range values (i.e. $4000-8000 \mu \mathrm{g} / \mathrm{L}$ at trough). As for many results emanating from specialized medicine laboratories, the clinical interpretation of TDM results is difficult for general practitioners. In the simplest case, the measurement of trough plasma level $\left(\mathrm{C}_{\min }\right)$ after 4 or more drug doses (i.e. once the steady-state is mostly achieved) along with the provision of reference therapeutic concentration ranges may be sufficient for ascertaining that patients are roughly exposed to appropriate drug concentrations. However, if the measured plasma concentration falls outside the target therapeutic range (i.e. either over- or under-exposure), the provision of specialized advice on how to interpret the concentration value and to adjust drug dosage in the patient brings an invaluable added-value over the mere delivery of a bare numerical result.

- Actually, concentration measurements are of no use for patients without thorough clinical interpretation in the conceptual framework of clinical pharmacokinetics [13]. TDM interpretation and drug dosage adaptation algorithms rely on the knowledge of the pharmacokinetic characteristics of the measured drug, such as the parameters describing its absorption $\left(K_{a}\right)$, its distribution volume $\left(V_{d}\right)$, its protein binding (\%)to circulating plasma proteins, its systemic hepatic and renal clearance (CL) etc. In addition, one must take into account the importance of their variabilities between patients and the factors influencing them in the specific clinical context of the patient. Factors most frequently affecting drug pharmacokinetics include anthropometric variables (age, gender, weight, height), physiopathological alterations of organs involved in drug disposition (renal and hepatic insufficiency), other co-morbidities (cancer, burns, cystic fibrosis, infections etc.), drug-drug interactions, food effects, special conditions (neonatology, paediatrics, pregnancy, obesity etc.) and finally (pharmaco)genetic factors that are being increasingly appreciated. There are further special situations such as dosage adjustments required during continuous extra-renal replacement therapy (i.e. hemodialysis or continuous veno-venous hemodiafiltration) in critically ill patients. Finally, drugs circulate in two forms in the blood: a fraction bound to plasma proteins (i.e. principally human serum albumin and/or $\alpha-1$ acid glycoprotein) and the remainder unbound (free) fraction. The TDM approach usually considers the measurement of total plasma concentration (i.e. the addition of free plus bound fractions in plasma), while only the free drug fraction is likely to diffuse into tissues and equilibrate with receptors and cells to exert the pharmacological activity. There are some special clinical conditions for which the measurement of free drug levels may be justified for some drugs, notably antiepileptics [14, 15].

Comprehensive studies of the pharmacokinetics and pharmacodynamics of a given drug are needed to decipher the relationships between drug dose, blood level (exposure), and therapeutic response and/or toxicity. Such studies are required during drug development and constitute an essential step for their rational use. Due to the complexity of real-life data, which confound the influences of multiple 
pharmacokinetic, environmental, genetic and drug-drug interactions into a single resulting phenotype, it has become suitable to develop models that can integrate all relevant determinants of treatment response. Population-based approaches currently represent the best way to characterize the pharmacokinetic-pharmacodynamic profile of a drug in a cohort of patients receiving it, and to capture the contribution of multiple genetic and non-genetic influences affecting drug levels [16, 17]. The basic concept of population modeling is to include patient data obtained from observational studies into non-linear mixed effects regression models to analyze data pooled over all the sampled individuals. Such pharmaco-statistical models incorporate both deterministic components (pharmacokinetic and pharmacodynamic models) and probabilistic components (statistical distribution of parameters across the population). For such purposes, several well established software programs have been developed, in particular NONMEM ${ }^{\circledR}$ (Non-linear Mixed Effect Modeling)[18]. Noteworthy, while having been developed for the purpose of research and drug development, such approaches have gained recognition of their clinical usefulness for patients' treatment, in particular as they allow Bayesian feed-back adjustment of dosage regimens, considered a key strategy for therapeutic drug monitoring [19]. Bayesian adjustment seems to provide the best possible approach, as illustrated e.g. for the targeted anticancer drug imatinib [20]. When drug levels are measured in samples taken at unselected times after drug intake, they must be interpreted with respect to actual dosing and sampling times, so that the measurement result can be exploited. This can be done through Bayesian maximum a posteriori approaches, yielding maximum likelihood estimates of a patient's individual concentration curve, integrated concentration exposure (AUC) or trough concentration level $\left(C_{\text {min }}\right)$. Such calculations have however to rely on appropriate computer tools [21] that aim at assisting practitioners in the interpretation of drug measurements.

Facing a given concentration result, the first question to ask is whether the measured drug levels is expected taking into account drug dosage and patient's characteristics, considering intrinsic population variability and aggregated population pharmacokinetic information. The "normality" or "expectedness" of a drug measurement result under a given dosage regimen is best assessed by comparison with reference percentiles derived from population pharmacokinetic analyses, which can be provided within routine TDM service (Figure 1). Such percentile curves have been elaborated based on data from large-scale studies conducted e.g. for several antiretrovirals [22-28], antibiotics [29, 30], antivirals [31], targeted anticancer agents [32, 33] etc.

Secondly, the question of "suitability" or "appropriateness" of actual exposure must be assessed considering therapeutic reference intervals. Such target ranges for exposure must also be based on population studies addressing concentration-effect relationships at the population level, such as established e.g. for the anti-HIV drug efavirenz [34, 35], or for voriconazole, an antifungal agent [36]. Target concentration ranges can be defined over the whole population, for subgroups of patients (Figure 1) or even for individual patients (e.g. based on virus susceptibility tests in the case of antiHIV therapies). 
Thirdly, the concentration result should also inform the prescriber upon how to modify the current dosage to reach the suitable target. The features of computer software developed to assist TDM allow dynamic pharmacokinetic modeling that can predict/simulate in silico the impact of any tested dose regimen on drug exposure in a given patient. In the future, such pieces of software should be integrated into the information systems of medical laboratories. In the long term, they might even become part of miniaturized measurement devices enabling point-of-care testing for blood concentration monitoring of critical drug agents, such as for instance last-resort antibiotics, antiretrovirals or targeted anticancer agents.

\section{TDM before the LC-MS/MS era}

Considerable progress in clinical pharmacokinetics and TDM has been made possible by the major advances in the field of bioanalysis over the last 25 years. In the 1980's, most clinical laboratories providing routine TDM services were using commercialized first generation immunoassays (Fluorescence Polarisation Immunoassays or FPIA, Enzyme-Multiplied Immunoassays Techniques or EMIT etc...) that allow the measurement of a single drug at a time. For drugs not measured by commercially available assays, laboratories developed in-house chromatographic methods using High performance liquid chromatography (HPLC) coupled to either Ultra-Violet (e.g. UV spectra captured on-line by Diode Array Detection), spectrofluorimetric or electrochemical detectors. The immunoassay approach suffers from the known limitations of antibodies-based methods (studied notably for. immunosuppressants, i.e. poor selectivity with respect to chemically related drug metabolites [37] and falsely elevated levels of tacrolimus [38, 39]), while the limited selectivity of HPLC notably coupled with UV detection frequently required prolonged chromatographic programs (i.e. up to 40-60 min per sample) that limited their practicability for routine application to real-time TDM.

Some 10 to 15 years ago, the LC-MS/MS technology raised and demonstrated impressive performances compared to the other bioanalytical methods available then. This prompted their rapid adoption in most medicine laboratory centres, despite the high costs of earlier instruments that made them prohibitive for some small laboratories. With prices going down, the access to LC-MS/MS technology has been democratized and this technology has now emerged as a key analytical tool for all modern clinical laboratories. High Performance and Ultra Performance Liquid Chromatography (HPLC and UPLC ${ }^{\circledR}$, respectively) coupled to MS/MS has revolutionized the way most drugs are now analysed, accelerating the pace of analytical developments and hence playing a major role for the current deployment of TDM.

In that context, excellent comprehensive articles have recently reviewed the current place of LCMS/MS in clinical laboratories both in general [2, 5] and specifically for routine TDM [1-4, 6]. The second part of the present article will highlight the general strengths, and also the current limitations, of LC-MS/MS technology specifically for the TDM approach. 


\section{The strength of LC-MS/MS for the TDM}

First, the unprecedented selectivity provided by tandem mass spectrometry makes it possible to simultaneously analyse a wide array of structurally unrelated analytes, and this within short analytical times (typically 5-7 min, including rinsing and re-equilibration steps) with both HPLC and UPLC ${ }^{\circledR}$. This approach has allowed the setting-up of numerous multiplex assays for the quantification of drugs from the same therapeutic class in a single chromatographic run. This multiplex approach has been applied notably for the simultaneous analysis of antiretroviral agents (in plasma and cells) [40-44], anti-HCV drugs[45], targeted anticancer agents [46], antimalarials [47], antifungals [48], new generation antiepileptics [49], (Figure 2), antibiotics such as several beta-lactams (carbapenems, penicillins, cephalosporins) altogether [50-53] or combined with other antibiotics classes including linezolid, ciprofloxacin, daptomycin and rifampicin [54-58]. Overall, the main advantages of this approach are: i) a unique sample extraction procedure and short analytical run; ii) time saving through the establishment of simultaneous calibration curves; iii) easy application to blood samples from patients receiving either single-drugs or combination regimens. This latter feature is particularly relevant for the analysis of triple therapies in HIV-infected patients at high risk of complex, reciprocal drug-drug interactions, or in case of difficult-to-treat epilepsy only controlled by antiepileptics combination (Figure 2). Moreover, iv) the results can be obtained on a daily basis, allowing the provision of TDM dosage adjustment advice within short time intervals. This is particularly important for the adjustment of therapy in hospital patients, e.g. intensive care patients with fluctuating pathophysiological conditions (renal and hepatic function). Finally, $v$ ) this multiplex approach allows the detection of other drugs from the same therapeutic class possibly present in samples, and this may also help to identify errors in drug prescription or administration. With regard to the possibility of detecting several drugs simultaneously, multiplex assays were applied to antimalarial drugs in pharmacokinetic field trials and revealed that at base-line, before the introduction of the trial drugs, about $74 \%$ and $50 \%$ of malaria patients in Tanzania and Cambodia, respectively, already had quantifiable levels of at least one antimalarial drug (i.e. prior to treatment initiation at the study center)[59, 60]. More generally, the improved analytical performances provided by current LC-MS/MS instruments allow the detection of extremely low drug levels in blood, never achieved so far. For drugs that are slowly eliminated from the human body, such an increased sensitivity has allowed to extend the time during which drugs can be detected in biological fluids, which in turn has led to update the half-life values of many therapeutic molecules. This is another example of the reciprocal interaction intrinsically linking clinical pharmacokinetics and bioanalytical methodologies.

As previously stated, the setting-up of assays by mass spectrometry has become greatly facilitated by the availability of stable isotopically-labelled Internal Standards (IS) (deuterium, ${ }^{13} \mathrm{C},{ }^{15} \mathrm{~N}$ ) that compensate for deleterious matrix effects variably affecting biological samples, which may otherwise compromise the accuracy of the analytical method. These IS are also important for the accurate quantification of analytes that are quantified simultaneously but cannot be chromatographically separated. For example, the antifungal drugs posaconazole and hydroxy-itraconazole have very similar chemical structures and are eluted at the same retention time [48]. The presence of their 
respective stable isotopically-labelled IS can correct for the potential reciprocal influence of both drugs at the ionisation step, which may be different in calibration samples (containing both drugs) and in patients plasma samples treated with only one antifungal agent. The use of isotopically-labelled IS also facilitates the measurement of drugs in other highly variable matrices such as urine, whose composition unlike plasma is not finely regulated by homeostasis and greatly varies with respect to patient hydration status, and food intake composition. For the assay of drugs in other biological matrices, such as dried blood spots, cerebrospinal fluid, cellular or tissue extracts, the use of isotopically-labelled IS insures that unidentified, possibly variable, matrix effect are compensated for.

More generally, as underscored by Adaway et al., the availability of LC-MS/MS equipments in clinical laboratories devoted to medium- to high-throughput analyses gives the user the flexibility to consolidate a range of additional esoteric or very specialized assays at very reasonable additional costs [2]

\section{The limitation of LC-MS/MS for the TDM}

Still, the LC-MS/MS technology has currently some limitations that are discussed herein. First, despite its unprecedented specificity, there are some reports of potentially false-positive results with LCMS/MS for drug analysis: yet, prevention strategies have been proposed to reduce the likelihood of such instances [61]. Second, the number of analytes that can be simultaneously assayed with multiplex methods is restricted to not more than one or two dozens. This is not because of the multiple mass transitions that have to be monitored simultaneously (i.e. Selected Reaction Monitoring, SRM), since most MS/MS instruments and especially the newer ones have the ability to scan a large number of $\mathrm{m} / \mathrm{z}$ transitions at very high frequency (up to $0.02 \mathrm{sec} / \mathrm{scan}$ ), not to mention the possibility to program several segments monitoring different $\mathrm{m} / \mathrm{z}$ transitions successively with respect to analytes' retention times. Rather, this limitation depends on the solubility of drugs that can be added in a biological matrix (generally plasma) for the preparation of multiplex calibration samples over large dynamic concentration ranges. This is especially a limitation when considering the multiplex analysis of lipophilic compounds that have relatively high plasma concentrations (i.e. anti-HIV drugs, antifungal drugs), taking into account that the percentage of non-matrix components (i.e. added solvents needed to solubilize analytes) should not be higher than $10 \%$ of the biological matrix of calibration samples.

Moreover, it has been argued that the tandem MS approach is so selective that the chromatographic step could be omitted, and the diluted samples extracts directly injected into the ionisation chamber. Based on our personal experience, we decidedly stand against this approach. Firstly, the chromatography (generally in the reverse phase mode) allows to eliminate early eluting, highly polar substances (sugar, inorganic salts, etc) present at very high concentrations in biological samples, and late (phospholipids) endogenous matrix components that would otherwise obscure - sometimes to an important extent - analyte's signals and that can hardly be compensated for by IS addition. Secondly, drug metabolites are present in patients' samples and some of them can be back-transformed into the parent drug through an in-source dissociation phenomenon, spuriously affecting parent drug levels in absence of chromatographic separation. This is observed for instance with the archetypal examples of voriconazole-NO, the CYP2C19-mediated oxidation product of the antifungal drug voriconazole 
[48], and raltegravir-glucuronide, the main metabolite of the HIV integrase inhibitor raltegravir [41, 62] [63]. These examples, among others, underscore the necessity of a chromatographic step to separate metabolites from parent drugs prior to the ionisation step.

Probably the major limitation of LC-MS/MS at present lies in the fact that, as they are designed, they constrain quantitative analyses to be performed batchwise rather than by random access as for most automated assays in clinical laboratories [2]. This lack of flexibility would a priori limit the application of LC-MS/MS to analytes for which a rapid TAT is not required [2]. This limitation can however be circumvented if the clinical laboratory is equipped with multiple LC-MS/MS instruments: one of our instruments remains specifically dedicated for the routine analysis of antibiotics for which reactive TDM interpretations are required for in-hospital patients. Typically, multiplex calibration curves are established on the morning, along with the first series of analysis of samples collected during the night. Any additional sample received in the laboratory during working hours is directly analysed along with a quality control sample. This makes antibiotics concentration results available for TDM interpretation almost in real-time, whith a delivery delay limited to some hours.

One recognized limitation of the mass spectrometry instruments is still their considerable acquisition costs. Nevertheless, several reports have demonstrated that the corresponding investment can in turn decrease overall analytical costs through increasing the productivity of laboratories. This has been observed e.g. when switching from immunoassays to mass spectrometry techniques for drug measurements [1, 64, 65]. While most mass spectrometry apparatus take at present a lot of laboratories room, some technological advances are being pursued to reduce the instrument's size, making them likely in the future to leave analytical laboratories and to be developed as point-of-care devices as recently demonstrated in the context of intraoperative tissue identification [66].

Finally, mass spectrometers manufacturers have constantly improved their instruments and software to render them more easily accessible to laboratory staff with possibly limited prior knowledge. Yet, mass spectrometry technology remains complex and quite demanding, and so far has not reached the same degree of automation as high-flux clinical chemistry using robotized analysers. A specialized staff of pharmacists, analytical engineers, and dedicated laboratory technicians with a high degree of analytical competence and skills is therefore still required for maintaining and fully exploiting the potential of LC-MS/MS platform. This critical mass of expertise may not be available in every hospital laboratory.

\section{Conclusion}

The various considerations addressed in this review have been inspired by our almost twenty-year of research and clinical experience with TDM. Certainly, the determination of the concentration of drugs in blood is neither the only nor the best way to monitor treatments; it should never replace the follow up of better suited clinical or biological biomarkers. Still for some therapeutic molecule, TDM has a rational role to pilot the treatment. TDM has not as sole objective to "verify the therapeutic activity" of 
a given drug, but rather, by enabling the adjustment of dosages, TDM aims to improve its efficacy and to prevent its toxicity. Pharmaceutical companies are however generally reluctant to include a detailed evaluation of individualization strategies in their drug development program and to consider TDM approaches that may complicate drug prescription to any extent: the one-pill-fits-all concept promoted by companies therefore stands in contrast to the growing importance of precision medicine, which implies minimizing chronic toxicities and managing drugs interactions at the individual patient level. Certainly, in the real-life situation, a number of patients may benefit from dosage adjustment for many of the drugs that they receive. The question is who among pharmaceutical companies, regulatory agencies or academic clinical pharmacologists should have to take the responsibility of the developing and implementing TDM approaches ? [13,67]. More generally, given the slowing-down of the pace of discovery of new clinically useful drugs, all strategies that can maximize the potential therapeutic benefit and safety of our current drug armamentarium have to be exploited. TDM can contribute meaningfully to the rational optimization and dosage individualization of specific critical new - and costly - drug treatments, notably antivirals, antibiotics and targeted anticancer agents, in the context of the emerging personalized medicine efforts. As "every patient is different", such optimization should allow administering in the routine setting "the right drug at the right dosage to the right patient".

With regards to analytical development, it is likely that in the future, technological innovations combining smaller measurement devices using alternate analytical approaches (plasmon resonance [68]), or possibly miniaturized mass spectrometry approaches, directly coupled to computer-assisted interpretation of concentration results may revolutionize TDM practices by enabling point-of-care testing for critical drug agents.

\section{Acknowledgement:}

This review article was made possible thanks to by the Swiss National Science Foundation (SNF) (grants $N^{\circ} 310030-138097$ and 32473-141234 to LAD) and within the frame of SNF-supported project NanoTera ISyPeM-2: "Developing therapeutic drug monitoring by designing a point-of-care system to measure drug concentration in blood samples and adjust dosage accordingly". www.nanotera.ch/projects/368.php, accessed January 20, 2016. 


\section{REFERENCES}

[1] F. Saint-Marcoux, [Some thoughts on the links between LC-MS/MS and therapeutic drug monitoring], Annales de biologie clinique, 73 (2015) 49-53.

[2] J.E. Adaway, B.G. Keevil, L.J. Owen, Liquid chromatography tandem mass spectrometry in the clinical laboratory, Annals of clinical biochemistry, 52 (2015) 18-38.

[3] J.E. Adaway, B.G. Keevil, Therapeutic drug monitoring and LC-MS/MS, Journal of Chromatography B, 883-884 (2012) 33-49.

[4] F. Saint-Marcoux, F.-L. Sauvage, P. Marquet, Current role of LC-MS in therapeutic drug monitoring, Anal Bioanal Chem, 388 (2007) 1327-1349.

[5] J.M. van den Ouweland, I.P. Kema, The role of liquid chromatography-tandem mass spectrometry in the clinical laboratory, Journal of chromatography B, 883-884 (2012) 18-32.

[6] N.J. Clarke, Mass Spectrometry in Precision Medicine: Phenotypic Measurements Alongside Pharmacogenomics, Clinical chemistry, 62 (2016) 70-76.

[7] A. Pascual, C. Csajka, T. Buclin, S. Bolay, J. Bille, T. Calandra, O. Marchetti, Challenging

recommended oral and intravenous voriconazole doses for improved efficacy and safety: population pharmacokinetics-based analysis of adult patients with invasive fungal infections, Clinical infectious diseases, 55 (2012) 381-390.

[8] T. Mercier, F. Tissot, C. Gardiol, N. Corti, S. Wehrli, M. Guidi, C. Csajka, T. Buclin, W. Couet, O. Marchetti, L.A. Decosterd, High-throughput hydrophilic interaction chromatography coupled to tandem mass spectrometry for the optimized quantification of the anti-Gram-negatives antibiotic colistin A/B and its pro-drug colistimethate, J Chromatogr A, 1369 (2014) 52-63.

[9] N. Widmer, C. Csajka, D. Werner, E. Grouzmann, L.A. Decosterd, C.B. Eap, J. Biollaz, T. Buclin, [Principles of therapeutic drug monitoring], Rev Med Suisse, 4 (2008) 1644-1648.

[10] M. van Luin, F.W. Wit, C. Smit, I.M. Rigter, E.J. Franssen, C. Richter, F. Kroon, F. de Wolf, D.M. Burger, Adherence to HIV therapeutic drug monitoring guidelines in The Netherlands, Therapeutic drug monitoring, 33 (2011) 32-39.

[11] N. Widmer, C. Bardin, E. Chatelut, A. Paci, J. Beijnen, D. Leveque, G. Veal, A. Astier, Review of therapeutic drug monitoring of anticancer drugs part two--targeted therapies, Eur J Cancer, 50 (2014) 2020-2036.

[12] N. Widmer, D. Werner, E. Grouzmann, C.B. Eap, O. Marchetti, A. Fayet, C. Csajka, L.A. Decosterd, T. Buclin, [Therapeutic drug monitoring: clinical practice], Rev Med Suisse, 4 (2008) 1649-1650, 16521660.

[13] T. Buclin, V. Gotta, A. Fuchs, N. Widmer, J. Aronson, Monitoring drug therapy, Br J Clin Pharmacol, 73 (2012) 917-923.

[14] A. Dasgupta, Usefulness of monitoring free (unbound) concentrations of therapeutic drugs in patient management, Clinica chimica acta, 377 (2007) 1-13.

[15] P.N. Patsalos, D.J. Berry, B.F. Bourgeois, J.C. Cloyd, T.A. Glauser, S.I. Johannessen, I.E. Leppik, T. Tomson, E. Perucca, Antiepileptic drugs--best practice guidelines for therapeutic drug monitoring: a position paper by the subcommission on therapeutic drug monitoring, ILAE Commission on Therapeutic Strategies, Epilepsia, 49 (2008) 1239-1276.

[16] J.S. Barrett, L. Labbe, M. Pfister, Application and impact of population pharmacokinetics in the assessment of antiretroviral pharmacotherapy, Clinical pharmacokinetics, 44 (2005) 591-625.

[17] L.B. Sheiner, T.M. Ludden, Population pharmacokinetics/dynamics, Annual review of pharmacology and toxicology, 32 (1992) 185-209.

[18] S.L. Beal, L.B. Sheiner, A.J. Boekmann, NONMEM User Guides (1989-2006) , locn Development Solutions, Ellicott City, MD, USA, DOI.

[19] M. Neely, R. Jelliffe, Practical therapeutic drug management in HIV-infected patients: use of population pharmacokinetic models supplemented by individualized Bayesian dose optimization, J Clin Pharmacol, 48 (2008) 1081-1091. 
[20] V. Gotta, N. Widmer, M. Montemurro, S. Leyvraz, A. Haouala, L.A. Decosterd, C. Csajka, T. Buclin, Therapeutic drug monitoring of imatinib: Bayesian and alternative methods to predict trough levels, Clinical pharmacokinetics, 51 (2012) 187-201.

[21] A. Fuchs, C. Csajka, Y. Thoma, T. Buclin, N. Widmer, Benchmarking therapeutic drug monitoring software: a review of available computer tools, Clinical pharmacokinetics, 52 (2013) 9-22.

[22] C. Csajka, C. Marzolini, K. Fattinger, L.A. Decosterd, J. Fellay, A. Telenti, J. Biollaz, T. Buclin, Population pharmacokinetics and effects of efavirenz in patients with human immunodeficiency virus infection, Clinical pharmacology and therapeutics, 73 (2003) 20-30.

[23] R. Lubomirov, M. Arab-Alameddine, M. Rotger, A. Fayet-Mello, R. Martinez, M. Guidi, J. di lulio, M. Cavassini, H.F. Gunthard, H. Furrer, C. Marzolini, E. Bernasconi, A. Calmy, T. Buclin, L.A. Decosterd, C. Csajka, A. Telenti, and the Swiss HIV Cohort Study, Pharmacogenetics-based population pharmacokinetic analysis of etravirine in HIV-1 infected individuals, Pharmacogenetics and genomics, 23 (2013) 9-18.

[24] R. Lubomirov, J. di lulio, A. Fayet, S. Colombo, R. Martinez, C. Marzolini, H. Furrer, P. Vernazza, A. Calmy, M. Cavassini, B. Ledergerber, K. Rentsch, P. Descombes, T. Buclin, L.A. Decosterd, C. Csajka, A. Telenti, ADME pharmacogenetics: investigation of the pharmacokinetics of the antiretroviral agent lopinavir coformulated with ritonavir, Pharmacogenetics and genomics, 20 (2010) 217-230.

[25] S. Colombo, T. Buclin, M. Cavassini, L.A. Decosterd, A. Telenti, J. Biollaz, C. Csajka, Population pharmacokinetics of atazanavir in patients with human immunodeficiency virus infection, Antimicrobial agents and chemotherapy, 50 (2006) 3801-3808.

[26] C. Csajka, C. Marzolini, K. Fattinger, L.A. Decosterd, A. Telenti, J. Biollaz, T. Buclin, Population pharmacokinetics of indinavir in patients infected with human immunodeficiency virus, Antimicrobial agents and chemotherapy, 48 (2004) 3226-3232.

[27] M. Arab-Alameddine, A. Fayet-Mello, R. Lubomirov, M. Neely, J. di lulio, A. Owen, M. Boffito, M. Cavassini, H.F. Gunthard, K. Rentsch, T. Buclin, M. Aouri, A. Telenti, L.A. Decosterd, M. Rotger, C. Csajka, and the Swiss HIV Cohort Study, Population pharmacokinetic analysis and pharmacogenetics of raltegravir in HIV-positive and healthy individuals, Antimicrobial agents and chemotherapy, 56 (2012) 2959-2966.

[28] M. Arab-Alameddine, R. Lubomirov, A. Fayet-Mello, M. Aouri, M. Rotger, T. Buclin, N. Widmer, M. Gatri, B. Ledergerber, K. Rentsch, M. Cavassini, A. Panchaud, M. Guidi, A. Telenti, L.A. Decosterd, C. Csajka, and the Swiss HIV Cohort Study, Population pharmacokinetic modelling and evaluation of different dosage regimens for darunavir and ritonavir in HIV-infected individuals, Journal of antimicrobial chemotherapy, 69 (2014) 2489-2498.

[29] F. Lamoth, T. Buclin, A. Pascual, S. Vora, S. Bolay, L.A. Decosterd, T. Calandra, O. Marchetti, High cefepime plasma concentrations and neurological toxicity in febrile neutropenic patients with mild impairment of renal function, Antimicrobial agents and chemotherapy, 54 (2010) 4360-4367.

[30] A. Fuchs, M. Guidi, E. Giannoni, D. Werner, T. Buclin, N. Widmer, C. Csajka, Population pharmacokinetic study of gentamicin in a large cohort of premature and term neonates, $\mathrm{Br} \mathrm{J}$ Clin Pharmacol, 78 (2014) 1090-1101.

[31] N. Perrottet, C. Csajka, M. Pascual, O. Manuel, F. Lamoth, P. Meylan, J.D. Aubert, J.P. Venetz, P. Soccal, L.A. Decosterd, J. Biollaz, T. Buclin, Population pharmacokinetics of ganciclovir in solid-organ transplant recipients receiving oral valganciclovir, Antimicrobial agents and chemotherapy, 53 (2009) 3017-3023.

[32] N. Widmer, L.A. Decosterd, C. Csajka, S. Leyvraz, M.A. Duchosal, A. Rosselet, B. Rochat, C.B. Eap, H. Henry, J. Biollaz, T. Buclin, Population pharmacokinetics of imatinib and the role of alpha-acid glycoprotein, Br J Clin Pharmacol, 62 (2006) 97-112.

[33] V. Gotta, S. Bouchet, N. Widmer, P. Schuld, L.A. Decosterd, T. Buclin, F.X. Mahon, C. Csajka, M. Molimard, Large-scale imatinib dose-concentration-effect study in CML patients under routine care conditions, Leuk Res, 38 (2014) 764-772.

[34] C. Marzolini, A. Telenti, L.A. Decosterd, G. Greub, J. Biollaz, T. Buclin, Efavirenz plasma levels can predict treatment failure and central nervous system side effects in HIV-1-infected patients, Aids, 15 (2001) 71-75. 
[35] A. Fayet Mello, T. Buclin, L.A. Decosterd, C. Delhumeau, J. di lulio, A. Fleurent, M.P. Schneider, M. Cavassini, A. Telenti, B. Hirschel, A. Calmy, Successful efavirenz dose reduction guided by therapeutic drug monitoring, Antiviral therapy, 16 (2011) 189-197.

[36] A. Pascual, T. Calandra, S. Bolay, T. Buclin, J. Bille, O. Marchetti, Voriconazole therapeutic drug monitoring in patients with invasive mycoses improves efficacy and safety outcomes, Clinical infectious diseases, 46 (2008) 201-211.

[37] W. Steimer, Performance and specificity of monoclonal immunoassays for cyclosporine monitoring: how specific is specific?, Clinical chemistry, 45 (1999) 371-381.

[38] J. Hermida, J.C. Tutor, Falsely increased blood tacrolimus concentrations using the ACMIA assay due to circulating endogenous antibodies in a liver transplant recipient: a tentative approach to obtaining reliable results, Therapeutic drug monitoring, 31 (2009) 269-272.

[39] L. Rostaing, O. Cointault, P. Marquet, A.G. Josse, M. Lavit, F. Saint-Marcoux, N. Kamar, Falsely elevated whole-blood tacrolimus concentrations in a kidney-transplant patient: potential hazards, Transplant international, 23 (2010) 227-230.

[40] S. Colombo, A. Beguin, A. Telenti, J. Biollaz, T. Buclin, B. Rochat, L.A. Decosterd, Intracellular measurements of anti-HIV drugs indinavir, amprenavir, saquinavir, ritonavir, nelfinavir, lopinavir, atazanavir, efavirenz and nevirapine in peripheral blood mononuclear cells by liquid chromatography coupled to tandem mass spectrometry, Journal of chromatography B, 819 (2005) 259-276.

[41] A. Fayet, A. Beguin, B. Zanolari, S. Cruchon, N. Guignard, A. Telenti, M. Cavassini, H.F. Gunthard, T. Buclin, J. Biollaz, B. Rochat, L.A. Decosterd, A LC-tandem MS assay for the simultaneous measurement of new antiretroviral agents: Raltegravir, maraviroc, darunavir, and etravirine, Journal of chromatography B, 877 (2009) 1057-1069.

[42] M. Aouri, A. Calmy, B. Hirschel, A. Telenti, T. Buclin, M. Cavassini, A. Rauch, L.A. Decosterd, A validated assay by liquid chromatography-tandem mass spectrometry for the simultaneous quantification of elvitegravir and rilpivirine in HIV positive patients, Journal of mass spectrometry, 48 (2013) 616-625.

[43] S. Colombo, T. Buclin, C. Franc, N. Guignard, M. Khonkarly, P.E. Tarr, B. Rochat, J. Biollaz, A. Telenti, L.A. Decosterd, M. Cavassini, Ritonavir-boosted atazanavir-lopinavir combination: a pharmacokinetic interaction study of total, unbound plasma and cellular exposures, Antiviral therapy, 11 (2006) 53-62.

[44] A. Fayet Mello, T. Buclin, C. Franc, S. Colombo, S. Cruchon, N. Guignard, J. Biollaz, A. Telenti, L.A. Decosterd, M. Cavassini, Cell disposition of raltegravir and newer antiretrovirals in HIV-infected patients: high inter-individual variability in raltegravir cellular penetration, Journal of antimicrobial chemotherapy, 66 (2011) 1573-1581.

[45] M. Aouri, D. Moradpour, M. Cavassini, T. Mercier, T. Buclin, C. Csajka, A. Telenti, A. Rauch, L.A. Decosterd, Multiplex liquid chromatography-tandem mass spectrometry assay for simultaneous therapeutic drug monitoring of ribavirin, boceprevir, and telaprevir, Antimicrobial agents and chemotherapy, 57 (2013) 3147-3158.

[46] A. Haouala, B. Zanolari, B. Rochat, M. Montemurro, K. Zaman, M.A. Duchosal, H.B. Ris, S. Leyvraz, N. Widmer, L.A. Decosterd, Therapeutic Drug Monitoring of the new targeted anticancer agents imatinib, nilotinib, dasatinib, sunitinib, sorafenib and lapatinib by LC tandem mass spectrometry, Journal of chromatography B, 877 (2009) 1982-1996.

[47] E.M. Hodel, B. Zanolari, T. Mercier, J. Biollaz, J. Keiser, P. Olliaro, B. Genton, L.A. Decosterd, A single LC-tandem mass spectrometry method for the simultaneous determination of 14 antimalarial drugs and their metabolites in human plasma, Journal of chromatography B, 877 (2009) 867-886. [48] L.A. Decosterd, B. Rochat, B. Pesse, T. Mercier, F. Tissot, N. Widmer, J. Bille, T. Calandra, B. Zanolari, O. Marchetti, Multiplex ultra-performance liquid chromatography-tandem mass spectrometry method for simultaneous quantification in human plasma of fluconazole, itraconazole, hydroxyitraconazole, posaconazole, voriconazole, voriconazole-N-oxide, anidulafungin, and caspofungin, Antimicrobial agents and chemotherapy, 54 (2010) 5303-5315. 
[49] L. Decosterd, T. Mercier, P. André, S. Bertholet, L. Rothuizen, T. Buclin, J. Novy, A. Rossetti, Multiplex mass spectrometry analysis of latest-generation antiepileptics: A clinically useful laboratory tool for improved real-time patients care, Epileptologie, 32 (2015) 85-89.

[50] F.B. Sime, M.S. Roberts, J.A. Roberts, T.A. Robertson, Simultaneous determination of seven betalactam antibiotics in human plasma for therapeutic drug monitoring and pharmacokinetic studies, Journal of chromatography B, 960 (2014) 134-144.

[51] M. Carlier, V. Stove, J.A. Roberts, E. Van de Velde, J.J. De Waele, A.G. Verstraete, Quantification of seven beta-lactam antibiotics and two beta-lactamase inhibitors in human plasma using a validated UPLC-MS/MS method, International journal of antimicrobial agents, 40 (2012) 416-422. [52] P. Colin, L. De Bock, H. T'Jollyn, K. Boussery, J. Van Bocxlaer, Development and validation of a fast and uniform approach to quantify beta-lactam antibiotics in human plasma by solid phase extraction-liquid chromatography-electrospray-tandem mass spectrometry, Talanta, 103 (2013) 285293.

[53] T. Ohmori, A. Suzuki, T. Niwa, H. Ushikoshi, K. Shirai, S. Yoshida, S. Ogura, Y. Itoh, Simultaneous determination of eight beta-lactam antibiotics in human serum by liquid chromatography-tandem mass spectrometry, Journal of chromatography B, 879 (2011) 1038-1042.

[54] J. Zander, B. Maier, A. Suhr, M. Zoller, L. Frey, D. Teupser, M. Vogeser, Quantification of piperacillin, tazobactam, cefepime, meropenem, ciprofloxacin and linezolid in serum using an isotope dilution UHPLC-MS/MS method with semi-automated sample preparation, Clin Chem Lab Med, 53 (2015) 781-791.

[55] R. Cazorla-Reyes, R. Romero-Gonzalez, A.G. Frenich, M.A. Rodriguez Maresca, J.L. Martinez Vidal, Simultaneous analysis of antibiotics in biological samples by ultra high performance liquid chromatography-tandem mass spectrometry, Journal of pharmaceutical and biomedical analysis, 89 (2014) 203-212.

[56] I.L. Tsai, H.Y. Sun, G.Y. Chen, S.W. Lin, C.H. Kuo, Simultaneous quantification of antimicrobial agents for multidrug-resistant bacterial infections in human plasma by ultra-high-pressure liquid chromatography-tandem mass spectrometry, Talanta, 116 (2013) 593-603.

[57] S. Barco, R. Bandettini, A. Maffia, G. Tripodi, E. Castagnola, G. Cangemi, Quantification of piperacillin, tazobactam, meropenem, ceftazidime, and linezolid in human plasma by liquid chromatography/tandem mass spectrometry, J Chemother, 27 (2015) 343-347.

[58] M. Gregoire, A.G. Leroy, R. Bouquie, D. Malandain, E. Dailly, D. Boutoille, C. Renaud, P. Jolliet, J. Caillon, G. Deslandes, Simultaneous determination of ceftaroline, daptomycin, linezolid and rifampicin concentrations in human plasma by on-line solid phase extraction coupled to highperformance liquid chromatography-tandem mass spectrometry, Journal of pharmaceutical and biomedical analysis, 118 (2016) 17-26.

[59] E.M. Hodel, A.M. Kabanywanyi, A. Malila, B. Zanolari, T. Mercier, H.P. Beck, T. Buclin, P. Olliaro, L.A. Decosterd, B. Genton, Residual antimalarials in malaria patients from Tanzania--implications on drug efficacy assessment and spread of parasite resistance, PloS one, 4 (2009) e8184.

[60] E.M. Hodel, B. Genton, B. Zanolari, T. Mercier, S. Duong, H.P. Beck, P. Olliaro, L.A. Decosterd, F. Ariey, Residual antimalarial concentrations before treatment in patients with malaria from Cambodia: indication of drug pressure, J Infect Dis, 202 (2010) 1088-1094.

[61] F.L. Sauvage, J.M. Gaulier, G. Lachatre, P. Marquet, Pitfalls and prevention strategies for liquid chromatography-tandem mass spectrometry in the selected reaction-monitoring mode for drug analysis, Clinical chemistry, 54 (2008) 1519-1527.

[62] M. Neely, L. Decosterd, A. Fayet, J.S. Lee, A. Margol, M. Kanani, J. di lulio, T. von Schoen-Angerer, R. Jelliffe, A. Calmy, Pharmacokinetics and pharmacogenomics of once-daily raltegravir and atazanavir in healthy volunteers, Antimicrobial agents and chemotherapy, 54 (2010) 4619-4625. [63] J.F. Jourdil, M. Bartoli, F. Stanke-Labesque, Lack of specificity for the analysis of raltegravir using online sample clean-up liquid chromatography-electrospray tandem mass spectrometry, Journal of chromatography B, 877 (2009) 3734-3738. 
[64] P.O. Hetu, R. Robitaille, B. Vinet, Successful and cost-efficient replacement of immunoassays by tandem mass spectrometry for the quantification of immunosuppressants in the clinical laboratory, Journal of chromatography B, 883-884 (2012) 95-101.

[65] P.J. Taylor, Therapeutic drug monitoring of immunosuppressant drugs by high-performance liquid chromatography-mass spectrometry, Therapeutic drug monitoring, 26 (2004) 215-219.

[66] J. Balog, L. Sasi-Szabo, J. Kinross, M.R. Lewis, L.J. Muirhead, K. Veselkov, R. Mirnezami, B. Dezso, L. Damjanovich, A. Darzi, J.K. Nicholson, Z. Takats, Intraoperative tissue identification using rapid evaporative ionization mass spectrometry, Science translational medicine, 5 (2013) 194ra193. [67] T. Buclin, N. Widmer, J. Biollaz, L.A. Decosterd, Who is in charge of assessing therapeutic drug monitoring? The case of imatinib, Lancet Oncol, 12 (2011) 9-11.

[68] G. Cappi, F.M. Spiga, Y. Moncada, A. Ferretti, M. Beyeler, M. Bianchessi, L. Decosterd, T. Buclin, C. Guiducci, Label-free detection of tobramycin in serum by transmission-localized surface plasmon resonance, Analytical chemistry, 87 (2015) 5278-5285.

[69] C. Robatel, L.A. Decosterd, J. Biollaz, P. Eckert, M.D. Schaller, T. Buclin, Pharmacokinetics and dosage adaptation of meropenem during continuous venovenous hemodiafiltration in critically ill patients, J Clin Pharmacol, 43 (2003) 1329-1340.

[70] F. Aucella, V. Lauriola, G. Vecchione, G.L. Tiscia, E. Grandone, Liquid chromatography-tandem mass spectrometry method as the golden standard for therapeutic drug monitoring in renal transplant, Journal of pharmaceutical and biomedical analysis, 86 (2013) 123-126.

[71] S.H. Song, S.H. Jun, K.U. Park, Y. Yoon, J.H. Lee, J.Q. Kim, J. Song, Simultaneous determination of first-line anti-tuberculosis drugs and their major metabolic ratios by liquid chromatography/tandem mass spectrometry, Rapid communications in mass spectrometry, 21 (2007) 1331-1338.

[72] J.S. Park, J.Y. Lee, Y.J. Lee, S.J. Kim, Y.J. Cho, H.I. Yoon, C.T. Lee, J. Song, J.H. Lee, Serum levels of anti-tuberculosis drugs and their effect on tuberculosis treatment outcome, Antimicrobial agents and chemotherapy,60(2015) 92-98.

[73] B. Wusk, G.A. Kullak-Ublick, C. Rammert, A. von Eckardstein, M. Fried, K.M. Rentsch, Therapeutic drug monitoring of thiopurine drugs in patients with inflammatory bowel disease or autoimmune hepatitis, European journal of gastroenterology \& hepatology, 16 (2004) 1407-1413.

[74] B. Wusk, G.A. Kullak-Ublick, C. Rammert, A. von Eckardstein, M. Fried, K.M. Rentsch, Thiopurine S-methyltransferase polymorphisms: efficient screening method for patients considering taking thiopurine drugs, Eur J Clin Pharmacol, 60 (2004) 5-10.

[75] E. Dahmane, T. Mercier, B. Zanolari, S. Cruchon, N. Guignard, T. Buclin, S. Leyvraz, K. Zaman, C. Csajka, L.A. Decosterd, An ultra performance liquid chromatography-tandem MS assay for tamoxifen metabolites profiling in plasma: first evidence of 4'-hydroxylated metabolites in breast cancer patients, Journal of chromatography B, 878 (2010) 3402-3414.

[76] N. Ansermot, M. Brawand-Amey, A. Kottelat, C.B. Eap, Fast quantification of ten psychotropic drugs and metabolites in human plasma by ultra-high performance liquid chromatography tandem mass spectrometry for therapeutic drug monitoring, J Chromatogr A, 1292 (2013) 160-172.

[77] N. Ansermot, M. Brawand-Amey, C.B. Eap, Simultaneous quantification of selective serotonin reuptake inhibitors and metabolites in human plasma by liquid chromatography-electrospray mass spectrometry for therapeutic drug monitoring, Journal of chromatography $B, 885-886$ (2012) 117130. 
Table 1: Clinical situations in which a drug blood concentration request can be considered

\begin{tabular}{|c|c|c|}
\hline Clinical situation & Objectives & Example \\
\hline $\begin{array}{l}\text { Less than expected clinical } \\
\text { response to therapy }\end{array}$ & $\begin{array}{l}\text { To distinguish true resistance } \\
\text { to pharmacological treatment } \\
\text { (i.e. despite sufficient drug } \\
\text { exposure) requiring treatment } \\
\text { change, from insufficient drug } \\
\text { exposure necessitating drug } \\
\text { dosage increase }\end{array}$ & $\begin{array}{l}\text { Seizures in epileptic patients } \\
\text { despite treatment with a given } \\
\text { antiepileptic agent }\end{array}$ \\
\hline $\begin{array}{l}\text { When over-dosage is } \\
\text { suspected, in the presence of } \\
\text { clinical signs of toxicity }\end{array}$ & $\begin{array}{l}\text { To identify toxicity due to drug } \\
\text { over-dosage, that may be } \\
\text { possibly confounded by the } \\
\text { underlying clinical conditions } \\
\text { (for instance in Intensive Care } \\
\text { Unit patients) }\end{array}$ & $\begin{array}{l}\text { TDM of the betalactam antibiotic } \\
\text { cefepime in the presence of } \\
\text { clinical signs such as } \\
\text { encephalopathy, convulsions, } \\
\text { nausea, vomiting, compatible } \\
\text { with intoxication. [29] }\end{array}$ \\
\hline $\begin{array}{l}\text { Known or suspected drug-drug } \\
\text { interaction problems }\end{array}$ & $\begin{array}{l}\text { To prevent adverse } \\
\text { consequences of the } \\
\text { interaction by drug dosage } \\
\text { adaptation }\end{array}$ & $\begin{array}{l}\text { TDM of the HIV integrase } \\
\text { inhibitor dolutegravir in the } \\
\text { presence of rifampicin, a potent } \\
\text { enzyme-inducing agent }\end{array}$ \\
\hline $\begin{array}{l}\text { Changes in the patho- } \\
\text { physiological state of patient } \\
\text { that may impair gastrointestinal, } \\
\text { hepatic or renal functions }\end{array}$ & $\begin{array}{l}\text { TDM-based drug dosage } \\
\text { adjustment to keep blood } \\
\text { levels within target therapeutic } \\
\text { window (i.e. notably above the } \\
\text { Minimum Inhibitory } \\
\text { Concentration of invasive } \\
\text { bacterial strains during e.g. at } \\
\text { least } 70 \% \text { of the dosing } \\
\text { interval) }\end{array}$ & $\begin{array}{l}\text { Septic shock patients receiving } \\
\text { meropenem [69](a carbapenem } \\
\text { antibiotic) while under } \\
\text { continuous extra-renal } \\
\text { replacement therapy } \\
\text { (haemodialysis, continous veno- } \\
\text { venous haemodiafiltration) }\end{array}$ \\
\hline $\begin{array}{l}\text { Special patients populations } \\
\text { (pregnancy, pediatrics) for } \\
\text { which there are limited } \\
\text { information available for drug } \\
\text { dosage adjustment }\end{array}$ & $\begin{array}{l}\text { To prevent under-dosage or } \\
\text { drug toxicity by TDM guided } \\
\text { drug dosage adaptation }\end{array}$ & $\begin{array}{l}\text { Optimization of antimicrobial } \\
\text { therapies with aminoglycosides } \\
\text { in neonates, to prevent renal or } \\
\text { auditory toxicities }\end{array}$ \\
\hline $\begin{array}{l}\text { Questionable adherence to } \\
\text { treatment }\end{array}$ & $\begin{array}{l}\text { TDM can only provide } \\
\text { information on short-term } \\
\text { compliance. Does not reflect } \\
\text { prolonged adherence to } \\
\text { therapy }\end{array}$ & $\begin{array}{l}\text { Undetectable antiretroviral drug } \\
\text { levels in plasma from HIV } \\
\text { patients with detectable HIV } \\
\text { RNA }\end{array}$ \\
\hline Clinical research & $\begin{array}{l}\text { To study drug } \\
\text { pharmacokinetics in humans } \\
\text { aiming at designing dosage } \\
\text { regimens for optimal drug } \\
\text { exposure }\end{array}$ & $\begin{array}{l}\text { Phase I, II, III or IV } \\
\text { pharmacokinetic studies, e.g. } \\
\text { comparison of once versus twice } \\
\text { daily administration of an anti-IV } \\
\text { agents, or quantification of the } \\
\text { consequences of a drug-drug } \\
\text { interaction }[43,62]\end{array}$ \\
\hline
\end{tabular}


Table 2. Therapeutic drug classes currently subjected to TDM in a tertiary hospital laboratory. All drugs are analysed by LC-MS/MS except a few drugs (indicated in italics) usually measured by immunoassays (aminoglycosides, glycopeptides) or by selective electrode or colorimetry (lithium) in the routine setting.

\begin{tabular}{|c|c|c|}
\hline Therapeutic classes & Examples & $\begin{array}{l}\text { Selected } \\
\text { References }\end{array}$ \\
\hline Immunosuppressants & $\begin{array}{l}\text { ciclosporin, tacrolimus, everolimus, sirolimus, } \\
\text { mycophenolate }\end{array}$ & {$[64,65,70]$} \\
\hline $\begin{array}{l}\text { Antiepileptics (earlier and newer } \\
\text { generations) }\end{array}$ & $\begin{array}{l}\text {-phenobarbital, phenytoin, carbamazepine, } \\
\text { valproate } \\
\text {-lamotrigine, topiramate, levetiracetam, } \\
\text { lacosamide, zonisamide }\end{array}$ & [49] \\
\hline Anti-HIV drugs & $\begin{array}{l}\text { darunavir, atazanavir, lopinavir, efavirenz, } \\
\text { nevirapine, rilpivirine, maraviroc, raltegravir, } \\
\text { elvitegravir, dolutegravir }\end{array}$ & [40-44] \\
\hline Antivirals & ribavirin, ganciclovir, acyclovir. & [45] \\
\hline Antifungals & $\begin{array}{l}\text { voriconazole, posaconazole, itraconazole, } \\
\text { hydroxy-itraconazole, fluconazole, caspofungin, } \\
\text { aniludafungin, micafungin, flucytocin }\end{array}$ & [48] \\
\hline $\begin{array}{l}\text { Antibiotics (early TDM drugs) } \\
\text { (aminoglycosides, glycopeptides) }\end{array}$ & $\begin{array}{l}\text { gentamicine, amikacine, tobramycine, } \\
\text { vancomycine, teicoplatin }\end{array}$ & \\
\hline $\begin{array}{l}\text { Antibiotics (recent TDM drugs) } \\
\text { carbapenems, betalactams, } \\
\text { lipopeptides, quinolones, others }\end{array}$ & $\begin{array}{l}\text {-imipenem, meropenem, cefepime , } \\
\text { piperacillin/tazobactam, amoxicillin, } \\
\text { flucloxacillin, ceftazidime, ceftriaxone, } \\
\text { cefuroxime, and } \\
\text { daptomycin, ciprofloxacin, levofloxacin, } \\
\text { linezolid, tygecyclin, etc... } \\
\text {-colistin ( + prodrug colistimethate) }\end{array}$ & $\begin{array}{c}{[50-53]} \\
{[54-58]} \\
{[8]}\end{array}$ \\
\hline Tuberculostatic drugs & $\begin{array}{l}\text {-isoniazide, rifampicine, pyrazinamide, } \\
\text { ethambutol }\end{array}$ & $\begin{array}{l}{[71]} \\
{[72]}\end{array}$ \\
\hline Antimalarials & quinine & [47] \\
\hline $\begin{array}{l}\text { Anticancer drugs (traditional } \\
\text { chemotherapies) }\end{array}$ & $\begin{array}{l}\text {-methotrexate, busulfan, mitotane, } \\
\text {-thiopurines* (azathioprine, mercaptopurine, } \\
\text { thioguanine) * formally, this is a phenotypic test } \\
\text { of the activity of thiopurine methyl-transferase }\end{array}$ & $\begin{array}{l}{[73]} \\
{[74]}\end{array}$ \\
\hline New targeted anticancer therapies & $\begin{array}{l}\text {-imatinib, nilotinib, dasatinib, bosutinib, } \\
\text { sunitinib, sorafenib, erlotinib, gefitinib, lapatinib, } \\
\text { verumafenib, regorafenib, etc } \\
\text {-tamoxifen /endoxifen. }\end{array}$ & $\begin{array}{l}{[46]} \\
{[75]}\end{array}$ \\
\hline $\begin{array}{l}\text { Psychotropics drugs } \\
\text { Antipsychotics, antidepressants etc. }\end{array}$ & $\begin{array}{l}\text {-lithium } \\
\text {-amisulpride, asenapine, mirtazapine, } \\
\text { iloperidone, olanzapine, paliperidone, } \\
\text { quetiapine, risperidone, etc... } \\
\text {-citalopram, fluoxetine, fluvoxamine, } \\
\text { paroxetine, sertraline, etc... }\end{array}$ & $\begin{array}{l}{[76]} \\
{[77]}\end{array}$ \\
\hline
\end{tabular}


Table 3. Principal precautions when performing blood sampling for TDM:

- The steady-state drug level has to be attained, i.e. the sample must be taken after 3-5 drug's half-lives post treatment initiation or after last dose change. Alternatively, pharmacokinetic accumulation must be accounted for during the interpretation of the result.

- Late sampling has to be privileged in general : ideally, trough $\left(\mathrm{C}_{\mathrm{min}}\right)$ levels are preferred (i.e. blood sampling just prior to the next dose). For aminoglycosides, (gentamicin, amikacin, tobramycin), $\mathrm{C}_{\text {min }}$ mainly correlates with toxicity while a "peak" sample is a better surrogate for bactericidal activity. Of note, in a number of instances (out-patient clinics) the blood sampling is done at the occasion of the medical visit, i.e. at unselected time after last drug intake. In that context, calculations derived from population pharmacokinetic models can be used to extrapolate $\mathrm{C}_{\text {min }}$ levels from concentration measured in samples taken at any time over the of dosing interval. New powerful - and user-friendy- interpretation tools are particularly helpful to that endeavour (see below and Figure 1). Still sampling after completion of the absorption phase should be systematically preferred.

- The blood collection must not be performed in patients on same arm that is used for i.v. administration, to avoid the risk of spuriously elevated drug levels emanating from the administered pharmaceutical preparation.

- Finally, and most importantly, recording a number of essential data is critical to correctly interpret the drug level measurement : i) date/time of blood sampling; ii) date/time of last drug intake or parenteral administration; iii) current dosage regimen (i.e. dose and frequency); iv) route of administration (intravenous, oral, etc.); v) date/time of treatment initiation, or last dosage change (to ascertain steady-state condition); vi) demographic data such as patient's age, gender, weight, height, special conditions (e.g. pregnancy); vii) relevant clinical information including indication for drug administration, kidney or liver dysfunction that may alter drug disposition, possibly extra-renal replacement therapy (hemodialysis, continuous veno-venous hemodiafiltration); and finally viii) reasons for TDM request (see Table 1). 
Figure 1: Typical example of concentration levels expected under a treatment of lopinavir $400 \mathrm{mg}$ twice a day, administered along with ritonavir boosting by $100 \mathrm{mg}$ twice a day. The black curve represents the average expectation and the grey zones encompass prediction percentiles according to time after dose intake at steady-state. The red vertical bars at trough time (12 h post-dose) depict the concentration targets defined for either treatment-naïve (dashed line) or experienced (heavy line) patients. While no upper limit is defined for the therapeutic range of lopinavir, we practically consider trough exposure over $8000 \mu \mathrm{g} / \mathrm{L}$ as of questionable usefulness.

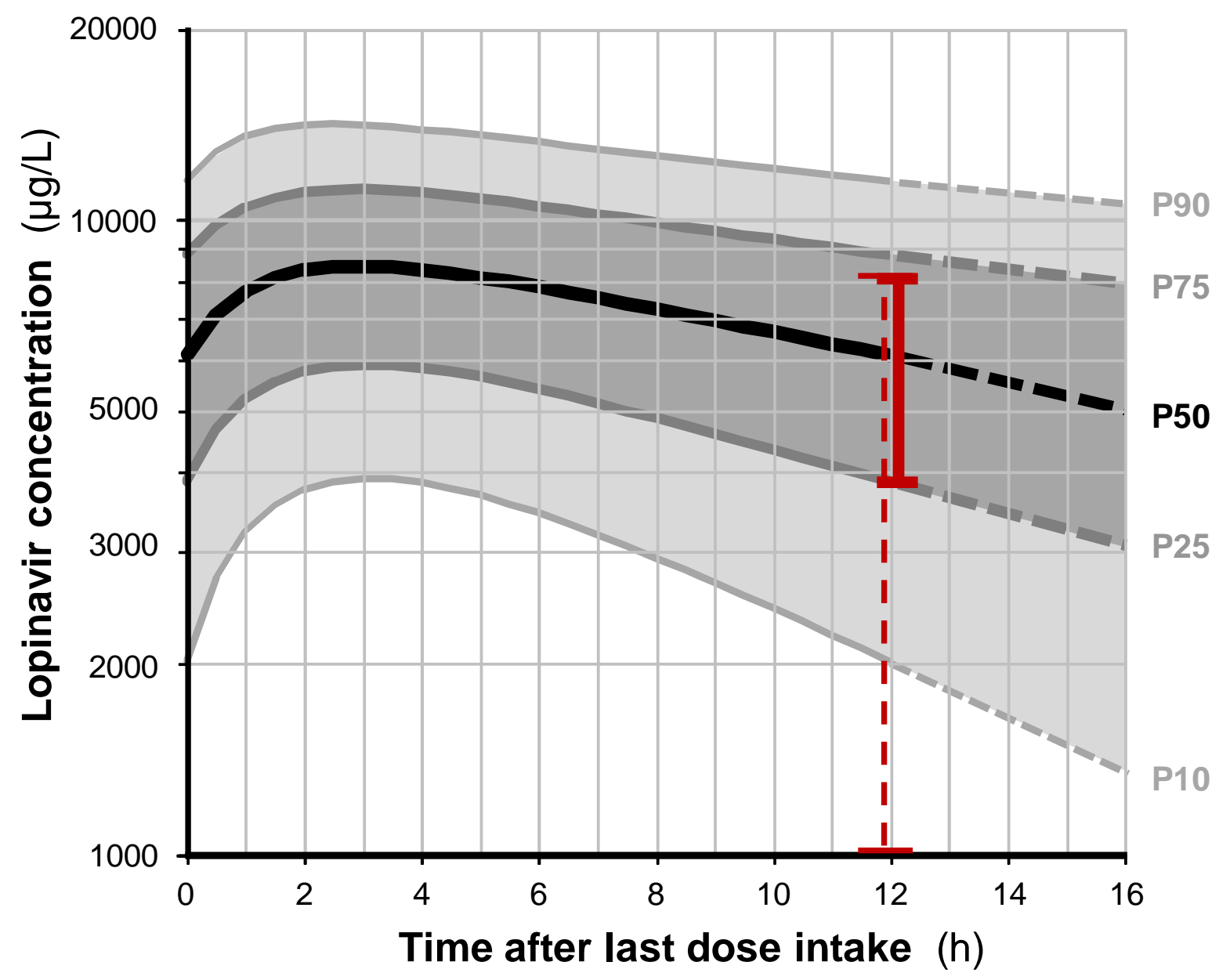


Figure 2: Application of multiplex LC-MS/MS for the real-time analyses of last generation antiepileptics combination. Chromatographic profile of a plasma from a woman (M.D.) receiving a regimen of the antiepileptic drugs levetiracetam, zonisamide and lamotrigine. The plasma levels in this patient are 16.2, 6.7 and $12.3 \mu \mathrm{g} / \mathrm{ml}$, respectively. The analytical run (including rinsing and re-equilibration time) lasts $6 \mathrm{~min}$. For simplification, the $\mathrm{m} / \mathrm{z}$ signals of the respective I.S. have been omitted. (see also [49])

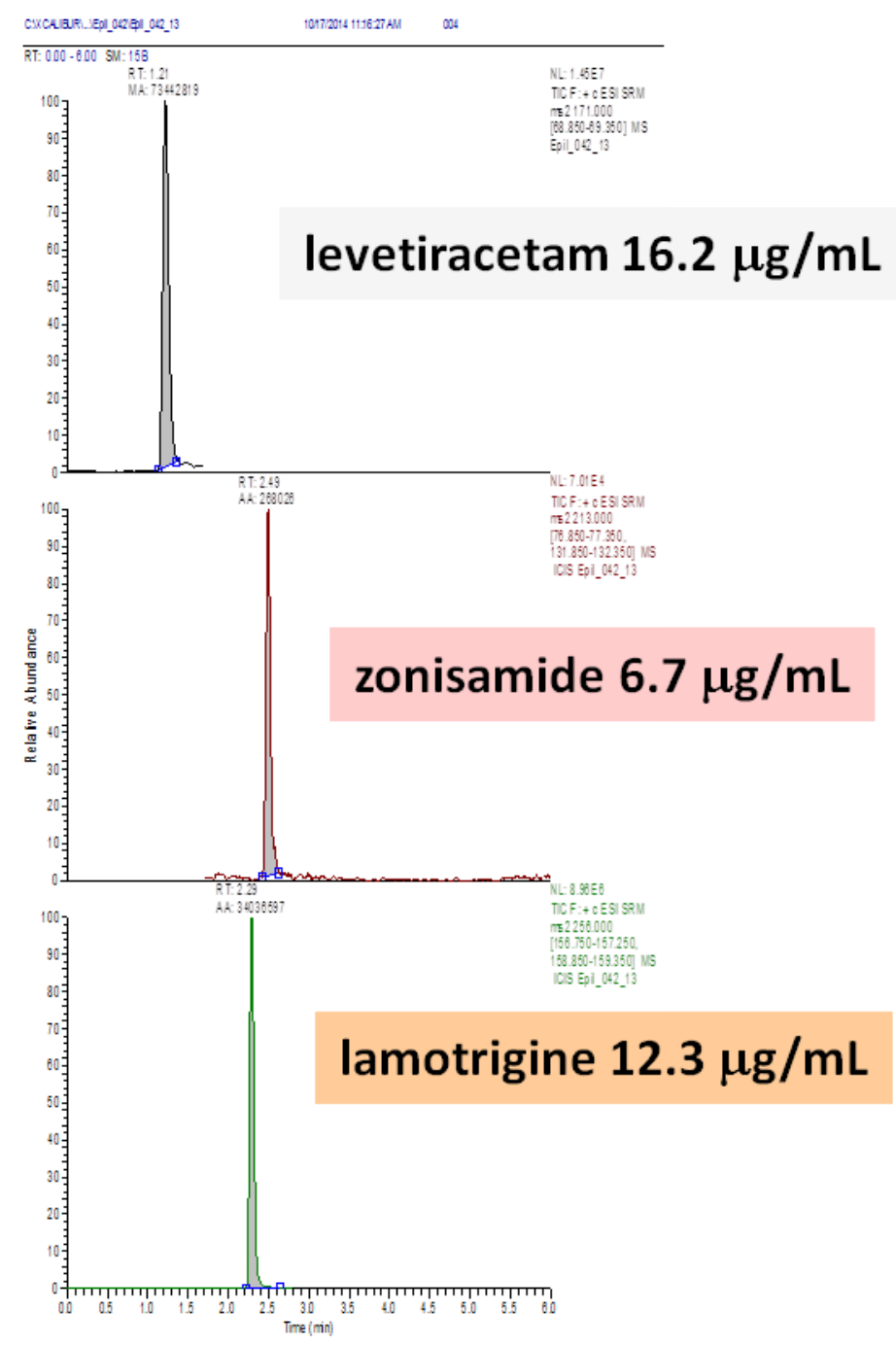

